\title{
Versatile Strategies for the Development of Wood-Based Functional Materials
}

\author{
Tobias Keplinger ${ }^{\mathrm{a}, \mathrm{b}}$, Marion Frey ${ }^{\mathrm{a}, \mathrm{b}}$, Ingo Burgert ${ }^{\mathrm{a}, \mathrm{b}}$ \\ ${ }^{a}$ Wood Materials Science, Institute for Building Materials (IfB), ETH Zürich, Stefano Franscini- \\ Platz 3, 8093 Zürich, Switzerland \\ ${ }^{\mathrm{b}}$ Applied Wood Materials Laboratory, EMPA-Swiss Federal Laboratories for Materials Science and \\ Technology, 8600 Dübendorf, Switzerland
}

\begin{abstract}
Reaching a sustainable society represents one of the key challenges of the $21^{\text {st }}$ century and wood as an abundant and renewable resource has the potential to serve as one of the main materials for this transition. In this regard different concepts for the development of novel wood-based functional materials, fabricated by the in situ formation of different materials systems are shown. Another focus is laid on densified cellulose composites, a novel material concept based on delignification and densification of wood, resulting in a high performance natural fibre reinforced composite material. This approach represents a promising alternative to common glass- and fiber reinforced composites but also to other manufacturing approaches such as 3D printing.
\end{abstract}

Keywords: functional wood materials, in situ functionalization, Raman spectroscopy imaging, densified cellulose scaffold; fiber reinforced composites

\section{INTRODUCTION}

Wood is characterized by a porous hierarchical structure across several length scales which results in excellent mechanical properties. This hierarchical structure, built up of aligned high strength cellulose fibers embedded in a pliant matrix of hemicelluloses and lignin, has been a great source of inspiration for the design of engineered composites ${ }^{2-4}$. Moreover, wood represents an ideal scaffold for inserting polymers or inorganic matter to develop high performance composites and hybrid materials ${ }^{5}$.

Numerous modifications/functionalizations have been developed in recent years, which on the one hand improve wood in its traditional application fields (e.g. increased dimensional stability, UV stability, improved flame retardancy) or on the other hand add new functionalities for novel hybrid materials including magnetic, electrical or stimuli-responsive properties $^{6-12}$.

Within the last years processing technologies in materials science have become more and more sophisticated providing excellent control of the micro- and nanostructure (e.g. 3D printing) of the respective manufactured materials. Nevertheless, it still remains highly challenging to upscale these structures and the therewith connected properties to the macroscale. Therefore, the functionalization of hierarchical natural materials (e.g. wood) is a promising alternative, as the hierarchical structure of wood can be directly utilized for up-scaling ${ }^{5}$.

Besides using natural wood for functionalization, delignification of wood while retaining the sophisticated hierarchical structure and the beneficial fibre directionality has caused significant interest as modifiable cellulose skeletons can be obtained. Previously used for the preparation of wood templated ceramics, this concept was applied for fabricating transparent wood and rather recently the wood inherent cellulose skeleton was used for the development of high performance densified bulk materials for sustainable engineering ${ }^{1,13,14}$.

In the following the main approaches for the development of wood-based hybrid materials via in situ formation of polymers and minerals with a specific focus on the analytical characterization on a cell wall level are shown. In addition, a specific focus will be put on discussing the concept of delignified wood as an important alternative to common fiber reinforced composites and to bottom up 3D printing approaches.

Bioinspiration, Biomimetics, and Bioreplication VIII, edited by Akhlesh Lakhtakia,

Proc. of SPIE Vol. 10593, 1059313 - () 2018 SPIE · CCC code:

0277-786X/18/\$18 - doi: 10.1117/12.2296537 


\section{MODULAR MODIFICATION APPROACHES}

Within the last years we have developed numerous modification/functionalization approaches for wood. The majority of them are based on the in situ formation of polymers respectively minerals within the bulk wood structure. In Figure 1, an overview of the various polymerization approaches developed is presented.

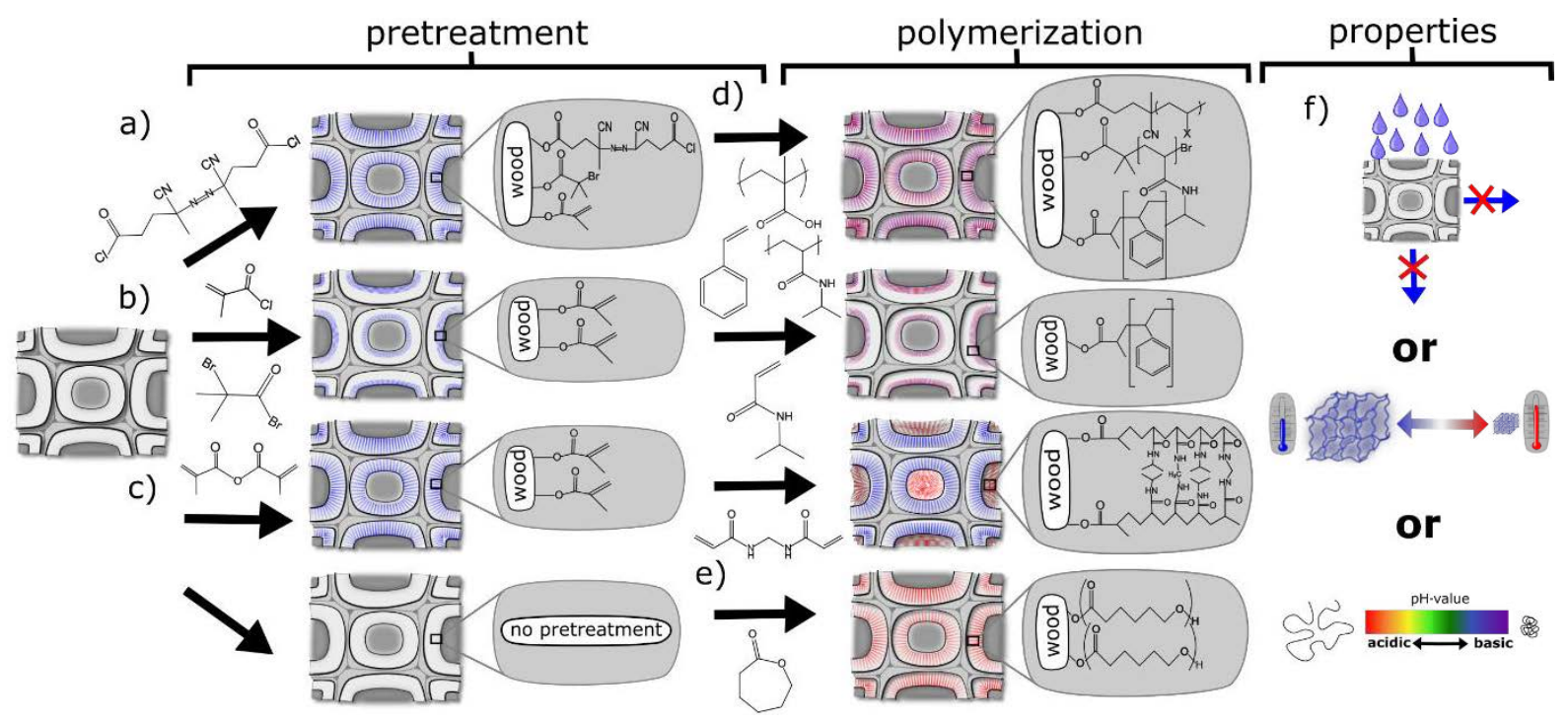

Figure 1. Scheme of modular modification approaches via polymers a) attachment of polymerization initiators to the wood inherent hydroxyl groups, b) reaction of hydroxyl groups with methacryloyl chloride, c) reaction of hydroxyl groups with methacrylic anhydride, d) in situ polymerization of different monomers such as styrene, methacrylic acid, Nisopropylacrylamid e) ring-opening polymerization of $\varepsilon$-caprolactone f) potential properties of the modified wood, for instance increased dimensional stability, responsiveness to temperature or $\mathrm{pH}$ changes

We have transferred various well-known polymerization techniques - including free radical polymerization (FRP), ringopening polymerization (ROP), and atom transfer radical polymerization (ATRP) - to the rather unusual substrate material wood. By these techniques, polymer chains were covalently attached to wood fibers. The versatility and potential of the approaches are twofold: by using various graft polymerization techniques, different monomers can be used and we can have better control of the final distribution of the polymer in the wood structure ${ }^{5,8,9,15,16}$.

In the following, two examples are presented in more detail. First, a simple approach which allows for a spatially controlled in situ formation of polymers and second, the development of a wood based stimuli responsive hybrid material based on the formation of PNIPAM hydrogel within the porous structure of wood.

\section{A modular approach with control of polymer distribution}

For wood functionalization approaches it is crucial to be able to control the spatial distribution of the functionalization within the bulk wood structure, as depending on the application needed, the distribution of the polymers within the wood structure must be adjusted.

For that in a first step, two bifunctional monomers with different reactivities (methacryloyl chloride and methacrylic anhydride) were used to introduce reactive methacryl groups into wood cell walls. The distribution of the methacryl groups can be controlled, based on the applied reaction protocol. By using these methacryl groups as anchor points for grafting, polymers can be inserted into the wood structure. Strikingly, depending on the methacryl precursor location, the spatial distribution of the polymer can be controlled (Figure 2). As a proof of concept, we have grafted polystyrene to the cell wall. For the case of methacryloyl chloride, the polymer was located mainly at the interface between cell wall and lumen, whereas in the case of methacrylic anhydride the polymer was distributed within the whole cell wall. For a detailed analysis of the different modification states within the wood cell wall, confocal Raman spectroscopy imaging was used, as it allows for a spatially resolved analysis with high chemical resolution (Figure 3$)^{9}$. 


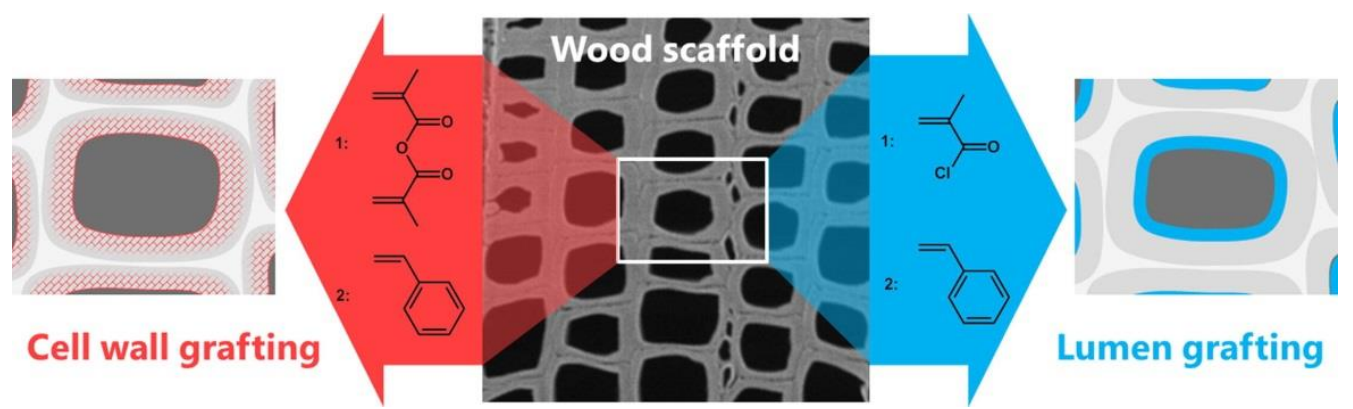

Figure 2. Scheme of the modification process: tunable functionalization of the wood cell wall with methacryl groups followed by grafting and in situ polymerization of styrene ${ }^{9}$.

The modular character of the here presented modification protocol allows for controlling the distribution of inserted monomers in the complex hierarchical structure of wood and can be varied depending on the needs of the final product. In order to generate high dimensional stability, hydrophobic polymers must be incorporated deep inside the wood cell wall. On the other hand wood modifications which target to make use of the microporous structure of wood, for example for membranes or filter applications, could profit from a preferential insertion of methacryl groups at the interface of cell wall and lumen as the related functional polymers can be attached in the right position?

\section{methacrylation}

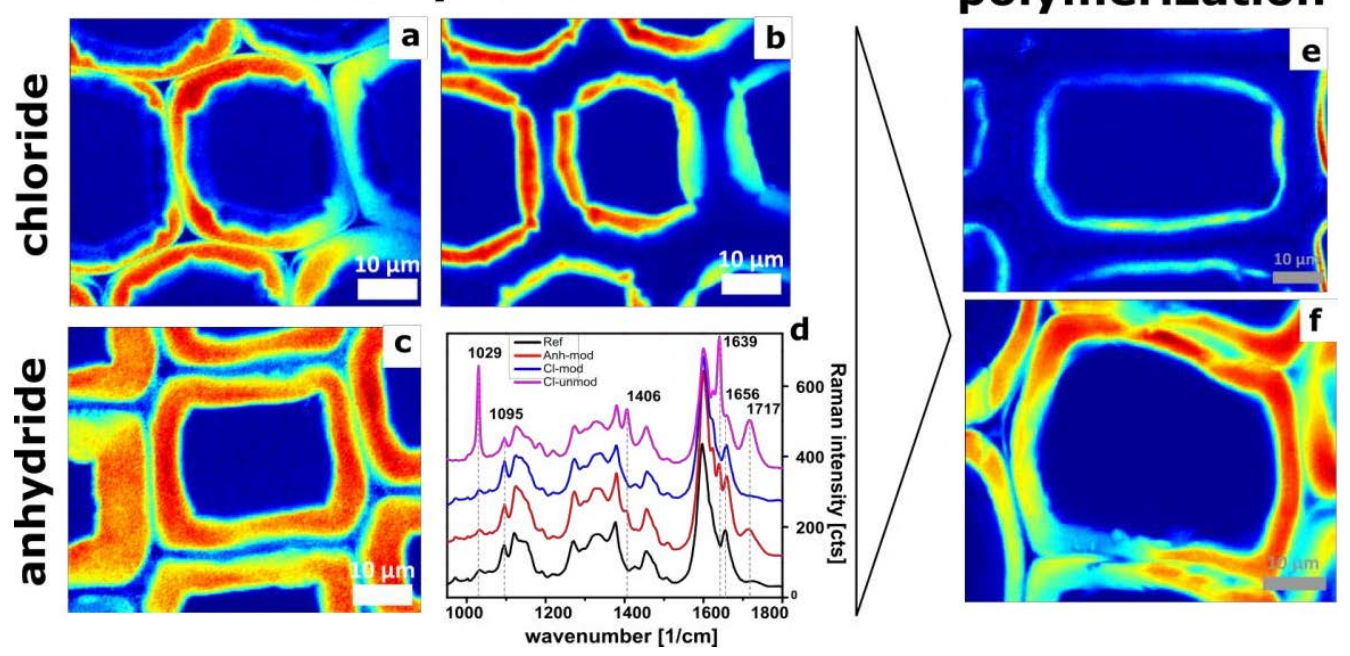

Figure 3. VCA (Vertex Component Analysis) of methacrylated and in situ polymerized wood samples; a) unmodified part of the wood cell wall after reaction with methacryloyl-chloride; b) modified part of the cell wall after reaction with methacryloyl chloride c) homogeneous distribution of methacryl groups within the wood cell wall after reaction with methacrylic anhydride; d) corresponding endmember spectra of the Raman images a-c; e) Raman images of the polymer distribution after pretreatment with methacryloyl chloride; f) polymer distribution after pretreatment with methacrylic anhydride?

\section{Stimuli-responsive wood based materials}

The development of new lightweight functional materials represents one of the main research directions in materials science. Thus we present here a simple two-step strategy for the formation of stimuli-responsive hydrogels within the hierarchical structure of wood, in order to create a new type of smart wood-based materials ${ }^{8}$.

In the first step of this modular functionalization, the wood cell walls were reacted with methacrylic anhydride. The anhydride attaches to the wood inherent OH-groups and the incorporated double bonds act as anchor points for the subsequent reaction of the hydrogel within the lumen of the wood cell walls. This ensures a stabilization of the hydrogel within the wood scaffold. Furthermore, by masking some of the hydroxyl functionalities, a certain hydrophobization of the cell wall occurs, which prevents hydrophilic monomers to enter the cell wall and constrains the monomers to the lumen $^{8}$. 
After the modification with methacryl groups a stimuli responsive hydrogel is formed inside the lumen of the wood cells by redox-initiated or UV-initiated polymerization. As a thermoresponsive polymer we have chosen crosslinked poly (Nisopropylacrylamide) (PNIPAM). PNIPAM is characterized by a lower critical solution temperature (LCST) of $32^{\circ} \mathrm{C}$. At this temperature, polymer chains undergo a transition from a well hydrated state to a hydrophobic collapsed state. This stimuli-responsive behavior within the wood structure has been investigated by Raman spectroscopy as this technique is not only suited for mapping the distribution of the constituents with high spatial resolution, but also it is highly sensitive to changes in the conformation of polymers. Thus, the structural changes of PNIPAM around the LCST can be studied. The conformational change can be measured by the changes in the $\mathrm{C}-\mathrm{H}$ stretching vibration. The $\mathrm{C}-\mathrm{H}$ region possesses a typical split into two peaks at $29451 / \mathrm{cm}$ and $29221 / \mathrm{cm}$ and the relative intensities of the two peaks are a measure of the hydration state of the isopropyl groups within the PNIPAM hydrogel. The hydrophilic-hydrophobic switch at the LCST can be therewith followed on the one hand by a drastic decrease of the water related peak around $32001 / \mathrm{cm}$ due to the dehydration of the hydrogel and on the other hand by change of the relative peak intensities of the double peak in the C$\mathrm{H}$ region (Figure 4$)^{8}$.
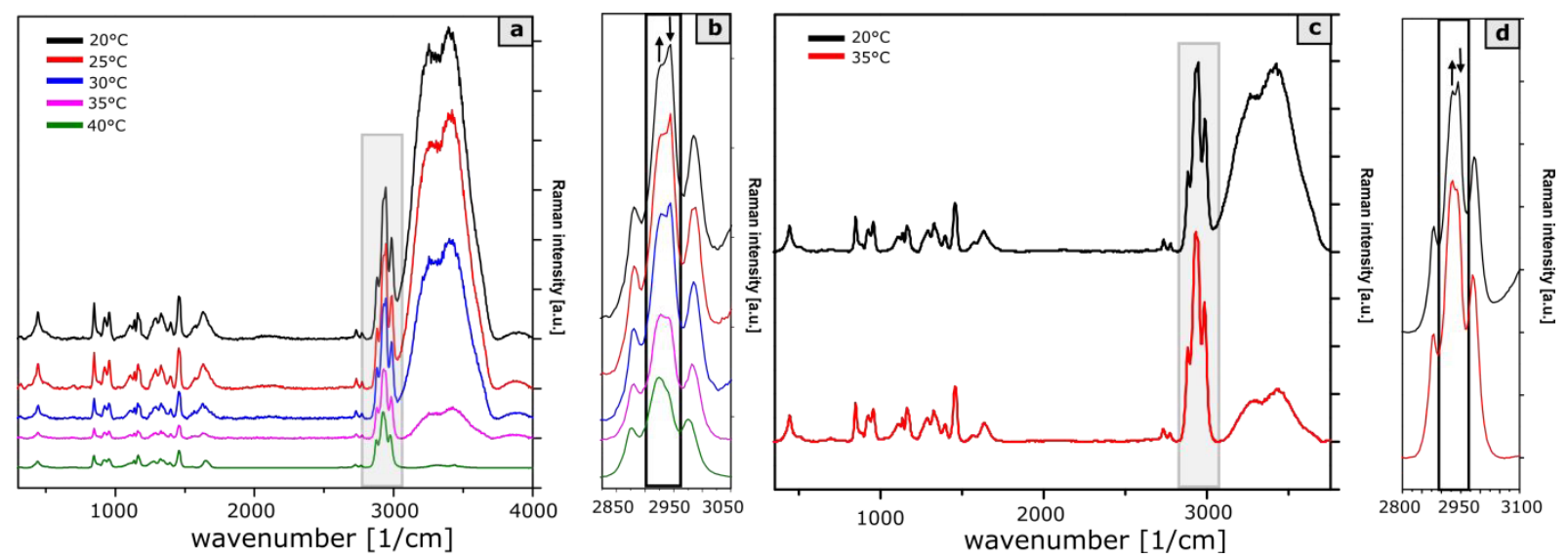

Figure 4. a) Raman spectra of pure PNIPAM hydrogel at different temperatures visualizing the drastic decrease of the water related $\mathrm{OH}$-band at $3200 \mathrm{1} / \mathrm{cm}$ above the LCST b) zoom into the $\mathrm{C}-\mathrm{H}$ stretching vibration revealing the change in the $\mathrm{C}-\mathrm{H}$ stretching region due to the changes in the hydration of the isopropyl groups of the hydrogel; c) average spectrum from the lumen of a hydrogel functionalized wood cell below and above the LCST and d) a zoom into the C-H stretching vibration which show similar spectral changes as the pure hydrogel ${ }^{8}$.

\section{Densified Cellulose Composites}

In materials science there is a growing interest in natural fiber reinforced composite materials due to an increasing demand for green, lightweight, high performance materials. The utilization of low cost natural fibers provides the possibility to replace traditional glass fiber composites. Nature itself provides a highly sophisticated natural fiber

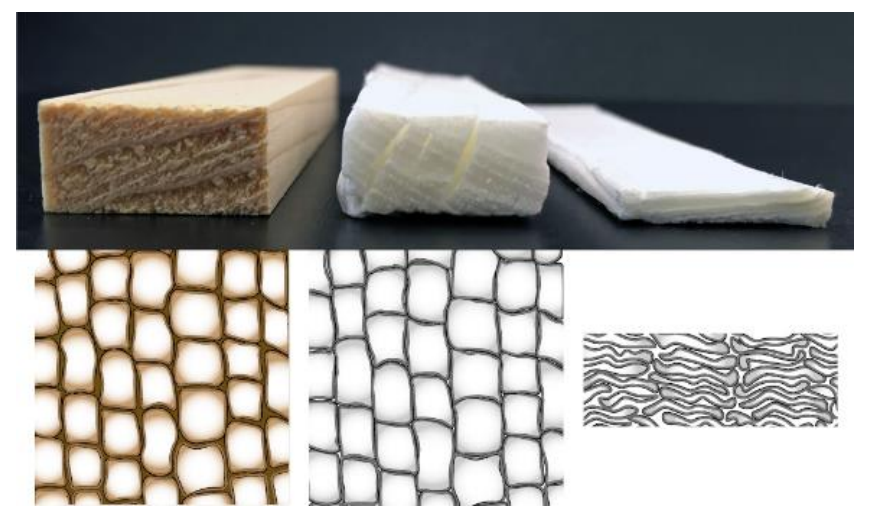

Figure 5. Illustration of the manufacturing process based on delignification followed by shear-assisted densification ${ }^{1}$ 
reinforced composites with wood as one famous example. The wood inherent cellulose framework represents an ideal scaffold for the development of hybrid composite materials.

Here we present the development of wood-templated cellulose fibre reinforced composites based on a novel material concept, which unifies delignification and densification of wood while retaining the hierarchical cellulose scaffold. The hereby obtained material possesses high stiffness and strength paired with high toughness when a resin is added, providing advantages compared to traditional natural fiber composites in terms of ease of production, fiber alignment and mechanical performance (Figure 5) ${ }^{1}$.

In a first step, wood samples were completely delignified and then the samples were densified with a newly developed densification procedure combining densification with lateral shearing. The shear assisted densification resulted in regular folding of the cells and reduced stress concentrations in the cell wall. The densified cellulose scaffolds possess highly desirable material properties such as high strength of up to $300 \mathrm{MPa}$ and a stiffness up to $40 \mathrm{GPa}$. Cellulose scaffolds can be shaped in wet state which allows the manufacturing of curved or twisted structures with perfectly oriented fibers. Additionally, density gradients can be created by varying the local pressure.

The scaffolds obtained by this novel processing method can be further modified by infiltration with different matrix systems. For the infiltration of the scaffolds, standard composite infiltration methods need to be optimized as the permeability, compared to textiles used for traditional fibre reinforced composites, is lower.

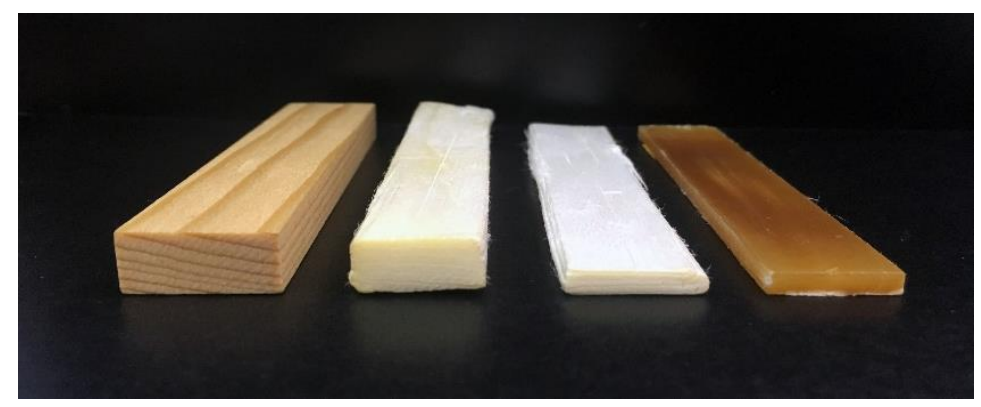

Figure 6. Image of natural wood; delignified cellulose scaffold; shear assisted densified cellulose scaffold; densified cellulose scaffold with epoxy matrix

\section{REFERENCES}

[1] M. Frey, D. Widner, J. S. Segmehl, K. Casdorff, T. Keplinger and I. Burgert, "Delignified and Densified Cellulose Bulk Materials with Excellent Tensile Properties for Sustainable Engineering," Acs Applied Materials \& Interfaces 10(5), 5030-5037 (2018)

[2] I. Burgert, "Exploring the micromechanical design of plant cell walls," Am. J. Bot. 93(10), 1391-1401 (2006)

[3] C. Sanchez, H. Arribart and M. M. G. Guille, "Biomimetism and bioinspiration as tools for the design of innovative materials and systems," Nature Materials 4(4), 277-288 (2005)

[4] U. G. K. Wegst, H. Bai, E. Saiz, A. P. Tomsia and R. O. Ritchie, "Bioinspired structural materials," Nature Materials 14(1), 23-36 (2015)

[5] I. Burgert, E. Cabane, C. Zollfrank and L. Berglund, "Bio-inspired functional wood-based materials - hybrids and replicates," International Materials Reviews 60(8), 431-450 (2015)

[6] E. Cabane, T. Keplinger, V. Merk, P. Hass and I. Burgert, "Renewable and Functional Wood Materials by Grafting Polymerization Within Cell Walls," ChemSusChem 7(4), 1020-1025 (2014)

[7] H. Z. Guo, D. Klose, Y. H. Hou, G. Jeschke and I. Burgert, "Highly Efficient UV Protection of the Biomaterial Wood by A Transparent TiO2/Ce Xerogel," Acs Applied Materials \& Interfaces 9(44), 39040-39047 (2017)

[8] T. Keplinger, E. Cabane, J. K. Berg, J. S. Segmehl, P. Bock and I. Burgert, "Smart Hierarchical Bio-Based Materials by Formation of Stimuli-Responsive Hydrogels inside the Microporous Structure of Wood," Adv. Mater. Interfaces 3(16), (2016)

[9] T. Keplinger, E. Cabane, M. Chanana, P. Hass, V. Merk, N. Gierlinger and I. Burgert, "A versatile strategy for grafting polymers to wood cell walls," Acta Biomaterialia (11), 256-263 (2015)

[10] V. Merk, M. Chanana, N. Gierlinger, A. M. Hirt and I. Burgert, "Hybrid Wood Materials with Magnetic Anisotropy Dictated by the Hierarchical Cell Structure," Acs Applied Materials \& Interfaces 6(12), 9760-9767 (2014) 
[11] V. Merk, M. Chanana, T. Keplinger, S. Gaan and I. Burgert, "Hybrid wood materials with improved fire retardance by bio-inspired mineralisation on the nano- and submicron level," Green Chemistry 17(3), 1423-1428 (2015)

[12] S. Trey, S. Jafarzadeh and M. Johansson, "In situ Polymerization of Polyaniline in Wood Veneers," Acs Applied Materials \& Interfaces 4(3), 1760-1769 (2012)

[13] D. Van Opdenbosch, G. Fritz-Popovski, O. Paris and C. Zollfrank, "Silica replication of the hierarchical structure of wood with nanometer precision," Journal of Materials Research 26(10), 1193-1202 (2011)

[14] Y. Y. Li, Q. L. Fu, S. Yu et al., "Optically Transparent Wood from a Nanoporous Cellulosic Template: Combining Functional and Structural Performance," Biomacromolecules, 17(4), 1358-1364 (2016).

[15] E. Cabane, T. Keplinger, T. Kunniger, V. Merk and I. Burgert, "Functional lignocellulosic materials prepared by ATRP from a wood scaffold," Scientific Reports 6, (2016)

[16] M. A. Ermeydan, E. Cabane, P. Hass, J. Koetz and I. Burgert, "Fully biodegradable modification of wood for improvement of dimensional stability and water absorption properties by poly(epsilon-caprolactone) grafting into the cell walls," Green Chemistry 16(6), 3313-3321 (2014) 